\title{
परमशिव एवं परब्रह्म
}

इन्दु शर्मा

सारांश

परमशिव एवं ब्रह्म दोनों ही भारतीय दर्शनों में परमसत्ता के रूप मे निरूपित हैं। एक का सम्बन्ध आगमों से है तथा दूसरे का सम्बन्ध वेदान्त से। अद्वैतवेदान्त में ब्रह्म की पूर्णतामूलक अवधारणा निषेधात्मक प्रतीत होती है क्योंकि उसमे कर्तृत्व व ज्ञातृत्व होते हुए भी स्वातन्त्र्य एवं बोध नहीं है। कर्तृत्व में स्वातन्त्र्य तथा विमर्श का और ज्ञातृत्व में बोध का एवं प्रकाश का सामरस्य आवश्यक है। तदपि यदि ब्रह्म में स्वातन्त्र्य को स्वीकार कर भी लिया जाए तो वह केवल अज्ञान का व्यावर्त्तक रूप ही होगा, साथ ही उसका स्वात्म विमर्शन नही होगा। इसके विपरीत परमशिव की ज्ञान क्रियामयता, प्रकाश विमर्शयता, चेतना, गतिमत्ता उसे एक विलक्षण परमसत्ता के रूप में सुस्थापित करती है। इस प्रकार "संविद्रब्म" संज्ञा जहाँ इसकी विशेषता को आभासित करती है, वहीं दूसरी ओर वेदान्तियों के ब्रह्म से इसका वैषम्य भी द्योतित कर देती है कि "वेदान्त का ब्रह्म जड़ब्रह्म" है।

कूट शब्द - शिव एवं ब्रह्म

प्रत्येक विचारशील मानव के अर्न्तमन में एक जिज्ञासा स्वभावतः ही पैदा होती है कि वह कौन है? कहाँ से आया है? यह जगत् क्या है? इस जगत का कर्ता या नियन्ता कौन है? वह कौन सा तत्त्व है जो हम सबका नियन्ता है? इन सब प्रश्नो के चिन्तन मे लीन मानव ने सर्वप्रथम अपने ही स्वरूप की खोज प्रारम्भ की। इस तत्त्व चिन्तन के प्रति जिज्ञासा की सर्वप्रथम स्पष्ट झलक हमें ऋग्वेद संहिता (ऋग्वेद संहिता, $1 / 164 / 46$ ) मे मिलती है। उपनिषद् काल मे भी यह जिज्ञासा वैसी ही बनी रही। जिसके द्वारा यह समस्त इन्द्रियाँ संचालित होती हैं? (केनोपनिषद्, 1)। वह कौन सा तत्त्व है जो परम सत् है। शब्दकल्पद्रुम मे "बृहि" धातु से बृद्धि अर्थ में ब्रह्मन् शब्द निष्पन्न किया गया है। "बृहत्वाद् बृह्मणत्वाद्वात्मैव ब्रह्मेति नीयते।" (राधाकान्तदेव, 1961)। इस वाक्य से ब्रह्म और आत्मा का अभेद सिद्ध होता है। वेद में परमतत्त्व, विप्र, प्रजापति, ब्रह्मादि भी ब्रह्मवाची हैं।

सर्वप्रथम आत्मन् शब्द प्राण या श्वास अर्थ में प्रयुक्त हुआ है (ऋग्वेद संहिता, 1/163/6)। उपनिषदों में प्राण शब्द को ही ब्रह्म का नाम दिया गया है (बृहदारण्यकोपनिषद्, 3/9/9)। "बृह" धातु से निष्पन्न ब्रह्म शब्द का पहला अर्थ संहिताओं में प्रार्थना या अन्न है। बह्म ब्रह्मणा कृण्वन्तः परिवत्सरीणम (ऋ0 सं0 7-103-8)। बह्म च तो जातवेदो नमश्च (ऋ0 सं०, 10-4-7)। आचार्य शंकर के अनुसार अपनी व्यापकता के कारण ही परम तत्त्व ब्रह्म कहलाता है (केनोपनिषद्, 1/5)। आचार्य शंकर ने ब्रह्म को सत्य और जगत को मिथ्या स्वीकार किया है (तैत्तिरीयोपनिषद्, $2 / 1 / 1)$ । श्रुति भी बृहि धातु से वृद्धि अर्थ में ब्रह्म शब्द की व्युत्पत्ति बताती है। बढा हुआ है तथा बढता है इसलिए परब्रह्म कहा जाता है (श्वेताश्वतरोपनिषद्,
$1 / 1)$ । अतः व्यापक होने के कारण ही ब्रह्म कहा जाता है (तैत्तिरीयोपनिषद्, 2/1/1)।

\section{अद्वैत वेदान्त में परब्रह्म का स्वरूप}

अद्वेत वेदान्त में ब्रह्म के स्वरूप का निरूपण इस प्रकार किया गया है "सच्चिदानन्द अद्वैत ब्रह्म ही वस्तु है अज्ञानादि समस्त जड़ समूह अवस्तु है। ब्रह्म ही नित्य वस्तु है। अन्य सब कुछ अनित्य है (तर्कवाचस्पतितारकनाथ, 1962) । "सत्य ज्ञानमन्मतं ब्रह्म" - वही ब्रह्म का स्वरूप लक्षण श्रुतियों द्वारा दिया गया है (तैत्तिरीयोपनिषद्, $2 / 1 / 1$ )। त्रिकालाबाधित वस्तु ही सत् है। उपनिषदों में ब्रह्म को पूर्ण सत्य के रूप मे स्वीकार किया गया है कि ब्रह्म के अतिरिक्त दूसरी कोई सत्ता नहीं है (बृहदारण्यकोपनिषद्, $3 / 9 / 9$ )। उस सत्य ब्रह्म का नाम भी सत् ही है (बृहदारण्यकोपनिषद्, $5 / 5 / 1$ )। उसके दर्शन, श्रवण और ज्ञान से समग्र जगत् का ज्ञान हो जाता है। अतः ब्रह्म ही अद्वेत एवं पूर्ण सत्ता है। यद्यपि ब्रह्म शब्द का प्रयोग सभी स्थलों पर अध्यात्मपरक नहीं है तथापि जहाँ भी इसका अध्यात्मपरक अर्थ है वहाँ सर्वोच्च सत्ता के रूप मे वर्णित है (श्रीमद्भगवद्गीता, 3-15, 4-24)। शंकराचार्य के अनुसार नामरूप के द्वारा अव्यक्त व मन से भी जिसकी रचना के स्वरूप का विचार नहीं हो सकता ऐसे जगत् की उत्पत्ति, स्थिति एवं नाश जिस सर्वज्ञ एवं सर्वशक्तिमान् कारण से होता है वह ब्रह्म है (शारीरकभाष्य, $1 / 1 / 2)$ । ब्रह्म की सत्ता को स्वीकार करते हुए स्पष्ट कहते हैं कि "ब्रह्मावसानोऽयं प्रतिषेधः नाभावावसानः" (शारीरकभाष्य, 3/2/22)। यद्यपि ब्रह्म को वाक् व मन से अतीत कहा है, परन्तु फिर भी वह अभाव रूप नहीं है। जो वस्तु नित्य है वही सत्य भी है, जो सत् है वह आत्मा ही है, उसी से समस्त जगत् की सत्ता है। वही परमार्थ सत् है। 
(छान्दोग्योपनिषद्, $6 / 8 / 7$ )। यदि ब्रह्म का तटस्थ लक्षण किया जाये तो उपनिषदों में इस प्रकार कहा गया है कि ब्रह्म ही जगत् का कारण है। उस एक ही ब्रह्म से यह समस्त नामरूपात्मक जगत् उत्पन्न होता है। वही इस जगत का निमित्त और उपादान कारण है। जैसे एक ही मृत्पिण्ड से अनेक पात्र बनते हैं और उसी में उनका लय होता है वैसे ही यह समस्त जगत् इस परम ब्रह्म से उत्पन्न होता है, उसी में स्थित है व उसी में लीन हो जाता है (छान्दोग्योपनिषद्, 6/1/4)।

\section{परब्रह्म की निमित्तोपादान कारणता}

आचार्य शंकर ने इस नामरूपात्मक जगत् प्रपंच का निमित्त और उपादान कारण परब्रह्म को ही माना है। जगत् की जन्म, स्थिति और लय का कारण बह्म ही है (शारीरकभाष्य, $1 / 4 / 13)$ । आकाशादि प्रपंचमय जगत् को कार्य तथा ब्रह्म को कारण कहा गया है (शारीरकभाष्य, 2/12)। मुण्डकोपनिषद् में ब्रह्म की निमित्तोपादानकारणता सिद्ध करने के लिए मकड़ी का दृष्टान्त दिया गया है जिस प्रकार मकड़ी अपना जाल बुनने के लिए किसी अन्य वस्तु की अपेक्षा नहीं करती उसी प्रकार वह परब्रह्म अपनी मायाशक्ति के द्वारा सम्पूर्ण सृष्टि को उत्पन्न करता है (बृहदारण्यकोपनिषद्, $1 / 17$ )। "येनाश्रुतं श्रुतं भवति " (छान्दोग्योपनिषद्, $6 / 1 / 13)$ । के द्वारा एक के जानने से अर्थात् ब्रह्म के जानने से सबका ज्ञान हो जाता है। आचार्य शंकर कहते हैं कि अन्य किसी अधिष्ठाता का अभाव होने से आत्मा कर्ता है अर्थात् निमित्त कारण है और अन्य उपादान का अभाव होने से आत्मा प्रकृति अथवा उपादान कारण भी है।"जन्माद्यस्य यतः" इस सूत्र का अर्थ करते हुए ब्रह्म का यही तटस्थ लक्षण दिया है। "यतो वा इमानि भूतानि जायन्ते" (तैत्तिरीयोपनिषद्, $3 / 1 / 1)$ । यह श्रुति इसमे प्रमाण है। इस प्रकार आचार्य शंकर श्रुति प्रमाण सहित जगत् की जन्म, स्थिति और लय का कारण ब्रह्म को ही मानते हैं (शारीरकभाष्य, 1/4/23)।

बह्म में प्रपंच की अभिन्न निमित्तोपादानकारणता विवर्तरूप है। सांख्यदर्शन में प्रकृति प्रपंच के प्रति परिणामी उपादान कारण है परन्तु अद्वैत मत में परिणाम और परिणामी का अभेद होने से बह्म भी परिणामी होगा और उसमें जन्मादि विकारों की प्रसक्ति होगी। इसलिए ब्रह्म में अविद्या से आकाश आदि प्रपंच के रूप से विवर्तोपादानता है। आकाशादि प्रपंच अपने कारणभूत परबह्म से पृथक् भी नहीं रह सकता है। जहाँ कही भी शंकराचार्य ने कार्य की वास्तविकता का खण्ड़न किया है कारण से भिन्न रूप में किया है। कहीं भी उन्होने व्यवहारिक जगत् को मिथ्या एवं
स्वप्न रूप नहीं कहा है (Radhakrishana, 1962)। यह नामरूपात्मक प्रपंच वास्तविक नहीं अपितु मायिक है माया या अविद्या से ही इस जगत् की सृष्टि हुई है। अद्वितीय ,अविकारी ब्रह्म ही माया के कारण नामरूपात्मक प्रपंच के रूप मे प्रतिभासित होता है किन्तु इस मायिक प्रतीति के कारण ब्रह्म के स्वरूप में कोई विकार या परिवर्तन नहीं होता, जैसे अविद्यावश रज्जु में सर्प का भ्रम हो जाने से रज्जु के स्वरूप में कोई परिवर्तन नहीं होता वैसे ही अविद्यावश ब्रह्म के जगत् स्वरूप में प्रतिभासित होने पर भी ब्रह्म के रूप में किसी प्रकार का विकार या परिवर्तन नहीं होता। अतः ब्रह्म को प्रपंच का विवर्तोपादानकारण मानने से ब्रह्म अथवा उसके धर्म में काई परिवर्तन नहीं होता। उत्पत्ति, स्थिति और प्रलयकाल में ब्रह्म अविकृत ही रहता है। ब्रह्म के रूप में कुछ भी विकृत न होकर आकाशादि प्रपंच की उत्पत्ति होती है (शारीरकभाष्य, 2/1/18)। अतः वेदान्त विवर्तवाद का ही समर्थक है।

\section{परमशिव का स्वरूप}

काश्मीर शैवदर्शन के अनुसार परमेश्वर या परमशिव ही एक मात्र परमसत्य है। वह शुद्ध प्रकाश स्वरूप है। वह पूर्ण चिद्रूप है। अतएव उसे चिति कहा गया है। चिति ही परासंवित् है (तंत्रालोकविवेक, भाग-3, पृ० 404)। शिव शब्द का शाब्दिक अर्थ है-मङ्गलकारी, कल्याणकारी, शुभ अथवा शिव शंकर। श्यति पापम, इस प्रकार इस शब्द की व्युत्पत्ति की जा सकती है (ईश्वरकृष्ण, 1970)।परमशिव का प्रकाश सूर्य के प्रकाश जैसा जड़ नहीं अपितु चैतन्य स्वरूप है अर्थात् परमशिव को अपनी सत्ता का अहं रूप में सतत आभास होता रहता है। शैवदर्शन के अनुसार यह प्रकाश विमर्शरूप है। प्रकाश और विमर्श एक दूसरे से सर्वथा अभिन्न है। एक के अभाव में दूसरे की कल्पना भी असम्भव है। इनमें अविनाभाव सम्बन्ध है (विज्ञानभैरव, पृ० 44)। प्रकाश आत्मा का स्वरूप है और विमर्श प्रकाशरूप परमात्मा के स्वरूप की प्रतीति है। वह विमर्श ही उसकी अपनी महेश्वरता की पूर्ण प्रतीति है (ईश्वरप्रत्यभिज्ञा, $1 / 8 / 11$ )। प्रकाश शिव रूप है और यह विमर्श शक्तिरूप है। शिव और शक्ति का नित्य सामरस्य ही परमशिव है। शिव के बिना शक्ति की सत्ता नहीं और शक्ति के बिना शिव स्फटिक आदि की भाँति जड़ तुल्य ही हो जायेगा, क्योंकि प्रकाशरूप होते हुए भी स्फटिक, मणि आदि को अपनी सत्ता की प्रतीति नहीं हाती (ईश्वरप्रत्यभिज्ञाविमर्शिनी, भाग-1, पृ० 198)। शिव-शक्ति के इसी अभेदभाव को लक्ष्य कर शिवदृष्टि में कहा गया है - 
न शिव: शक्तिरहितो न शक्तिर्य्यतिरेकिणी । शिव: शक्तस्तथा भावानिच्छया कर्तुमीहते // शक्तिशक्तिमतोर्भेदः शैवे जातु न वर्ण्यते (शिवदृष्टि, 3/2-3)।

शक्ति स्वभाव से " शक्त " होने पर ही शिव कर्तृत्व पद का अधिकारी होता है। शक्ति आत्मारूपी परमशिव का विमर्श है और इस विमर्श से ही वह "कर्तुम्" "अकर्तुम्" -"अन्यथाकर्तुम्" स्वभावी होता है (ईश्वरप्रत्यभिज्ञाविमर्शिनी, भाग-1, पृ० 205)। अपने उक्त विमर्श से सब कुछ कर सकने के कारण परमशिव पूर्ण स्वतन्त्र है। स्वतन्त्र परमशिव की इच्छाशक्ति ही इसका स्वातन्त्र्य कहलाता है (मालिनीविजयवार्तिक, 1/87)। इसी में ज्ञान शक्ति और क्रिया शक्ति सदैव अभेदरूप में स्फुरित होती रहती है (ईश्वरप्रत्यभिज्ञाविमर्शिनी, $1 / 8 / 11)$ । ज्ञान और क्रिया वस्तुतः एक पारमेश्वरी इच्छा का ही उत्तरोत्तर विकास है। परमेश्वर की इस चिकीर्षारूप इच्छा में सब कुछ अन्तर्भूत है और वह सब वहाँ अभेदरूप से ही अवस्थित है (ईश्वरप्रत्यभिज्ञाविमर्शिनी, भाग-1, पृ० 181)। इसी कारण " शिवदृष्टि " में कहा गया है कि आत्मा अपनी स्वतन्त्र इच्छा से ही शिव से लेकर पृथ्वी पर्यन्त सर्वत्र अभेदभाव से स्फुरित है (शिवदृष्टि, 1/2)। काश्मीर शैवदर्शन में छत्तीस तत्त्वों को स्वीकार किया गया है। शिव से लेकर पृथ्वी तक छत्तीस तत्त्वों में अभेदरूपता से स्फुरित आत्मा का इच्छा प्रसार ही उसका विश्वात्मक रूप है। इसी को शैवागम में विमर्श कहते हैं। क्योंकि विमर्श परमशिव की शक्ति है और उसकी शक्ति का स्फार ही नानारूपात्मक विश्व है (ईश्वरप्रत्यभिज्ञाविमर्शिनी, भाग-2, पृ० 42)। इस प्रकार परमशिव विश्वोत्तीर्ण और विश्वात्मक द्विविध स्वरूप है (तंत्रालोकविवेक, भाग-1, पृ० 105)। काश्मीर शैवदर्शन के विश्वोत्तीर्ण और विश्वात्मक स्वरूप वाले परमशिव का ऋग्वेद के पुरूष सूक्त मे निरूपित स्वरूप वाले " परम पुरूष" के साथ पूर्ण सामंजस्य है (ऋ0 सं0, 10/90)।

\section{परमशिव का आभास}

यह सम्पूर्ण स्फार परमेश्वर परमशिव की इच्छा से ही होता है। इसी कारण आचार्य क्षेमराज ने प्रत्यभिज्ञाहृदयम् में चिदात्मा को ही विश्व उल्लास का कारण बताया है (प्रत्यभिज्ञाहृदयम्, सूत्र-1)। चिति अपनी स्वतन्त्र इच्छा से आत्म - भित्ति पर अर्थात् अपनी चिद्रूपता (आश्रय) के अन्तर्गत ही अभेदरूप से विश्व को उन्मीलित करती है (प्रत्यभिज्ञाहृदयम, सूत्र-2)। अतः परमेश्वर स्वयं ही उपादान कारण है। चिदात्मा परमेश्वर की असंख्य शक्तियों मे से पाँच शक्तियाँ मुख्य मानी गयी हैं-चित्, आनन्द, इच्छा, ज्ञान ,और क्रिया (तंत्रसार, पृ० 6)। इन्ही शक्तियों के द्वारा
परमशिव पहले अभेद दशा से भेदाभेद दशा में तथा भेदाभेद दशा से भेद दशा में स्वयं अवतरित होते हैं और सम्पूर्ण विश्व को प्रकाशित करते हैं। दर्पणनगरन्याय की भाँति विश्व और परम शिव में अभेद है, भेद नहीं। यह सम्पूर्ण विश्व उनसे अभिन्न होते हुए भी भिन्नवत् अवभासित होता है इसलिए समस्त पदार्थ आभासरूप कहे गये हैं। क्योंकि ये परमशिव के द्वारा आभासित किए गए हैं (तंत्रालोकविवेक, भाग-2, पृ० 29, 30)। विश्वात्मकरूप में अपनी परमेश्वरता (शक्ति) का यह आभास ही परमेश्वर का स्वात्मविनोद है और इसकी यह आभास-लीला (विश्व लीला) ही जीवों के लिए परमशिव की सत्ता की द्योतक है (ईश्वरप्रत्यभिज्ञाविमर्शिनी, भाग-1, पृ० 195)।

\section{परमशिव एवं परब्रह्म का विश्लेषण}

पूर्णता की दृष्टि से काश्मीर शैवदर्शन ही अधिक समीचीन है। इसका मुख्य कारण है परमेश्वर परमशिव का स्वातन्त्र्य। अद्वेतवेदान्त में ब्रह्ममूलक धारणा ज्ञानमार्गी है क्योंकि यहाँ कैवल्य का निश्चय मुख्यतः ज्ञान के माध्यम से होता है, यही दृष्टिकोण निर्वाण को स्वीकार करने वालों का भी है। परन्तु काश्मीर शैवदर्शन में परमेश्वर परमशिव अप्रतिहत इच्छा प्रसार वाले हैं। परमशिव की इच्छाशक्ति ही शिवाद्वयवादी दृष्टिकोण को पूर्णता प्रदान करती हैं (रस्तोगी, 2002)। आगमिक दर्शन में स्वतन्त्रता और पूर्णत्व के प्रत्यय मूलभूत हैं। यद्यपि वेदान्त में स्वतन्त्रता तथा पूर्णता ब्रह्म की स्वरूपभूत विशेषताएँ या स्वभाव स्वीकार किए गए हैं फिर भी उसका दृष्टिकोण नकारात्मक ही रहा है। यहाँ ब्रह्म की पूर्णता इस बात की द्योतक है कि यह भवनात्मकता से स्वतन्त्र है, किन्तु यहाँ पर यह प्रश्न होता है कि वेदान्ती जिस तरह की पूर्णता को स्वीकार करते हैं (अर्थात् व्यावहारिक रूप में जगत् एवं आत्मा की सत्ता को अलग-अलग स्वीकार करना) उसे पूर्णता का संकेतक माना जा सकता है? अद्वेतवेदान्त का अद्वेतवाद सभी को समन्वित करने वाला भी नहीं हो सकता क्योंकि उसे माया को वास्तविक मानने में संकोच होगा। वेदान्त का अद्वैत सभी से भिन्न है क्योंकि वह ब्रह्मेतर सभी के अपसारण पर निर्भर करता है। यह अन्यों से पृथक्कृत शुद्धता (विश्वोत्तीर्णता) का प्रतिपादक है। यहाँ ज्ञान एवं अज्ञान या सत् और असत् परस्पर विरूद्ध माने गये हैं। वेदान्त में ब्रह्म को सच्चिदानन्द कहा गया, फिर भी उस पर यह आक्षेप लगता है कि वह स्वात्मचेतना एवं समरसता से रहित है। अब यहाँ प्रश्न उठता है कि क्या सत्, चित् एवं आनन्द प्रत्ययमूलक अर्थो में भेद है? क्योंकि जो चित् है, निश्चित ही उसे स्वात्म चेतना रूप होना चाहिए और जो आनन्दात्मा है, उसे समरसता या 
समन्वय होना चाहिए। यहाँ सत्, चित् एवं आनन्द तीनों एकात्मक रूप से ब्रह्म का संकेतन करते हैं किन्तु यहाँ क्रिया या स्वातन्त्र्य को स्वीकार नहीं किया गया है। वेदान्त में चित् का स्वातन्त्र्य स्वीकार नहीं है, चिद्रूपा शक्ति यहाँ सुप्त है। इसीलिए ऐश्वर्य उपाधिगत तथा आगन्तुक माना गया है। आगम सम्मत चित्स्वरूप में ऐश्वर्य उपाधिमूलक न होकर स्वाभाविक है। उसी का नामान्तर स्वातन्त्र्य है। जिसकी स्वतन्त्र सत्ता होती है क्रिया में उसी का स्वातन्त्र्य होता है (अजडप्रमातृसिद्धि, श्लोक-23) और स्वतन्त्र सत्ता परमेश्वर परमशिव है (तंत्रालोक, 1/222)।

\section{निष्कर्ष}

अद्वैतवेदान्त में ब्रह्म की पूर्णतामूलक धारणा निषेधात्मक है। उसमें स्वातन्त्र्य तथा बोध नहीं है। कर्तृत्व में स्वातन्त्र्य एवं विमर्श का और ज्ञातृत्व में बोध एवं प्रकाश का सामरस्य आवश्यक है। किसी तरह उसके स्वातन्त्र्य को मान भी लिया जाये तो वह केवल अज्ञान के व्यावर्त्तक रूप में होगा साथ ही उसका स्वात्म विमर्शन नहीं होगा।

इस प्रकार ऐसा प्रतीत होता है कि अद्वेतवेदान्त का दृष्टिकोण निषेधात्मक ही है। वेदान्त की इस बात की प्रशंसा की जानी चाहिए कि वह स्पष्ट रूप से जगत् का निषेध करता है। शंकर ब्रह्म को सत्य और माया को अनिर्वचनीय कहते हैं। इसलिए वाक्यों द्वारा जितना अद्वैत भाव दिखाने की चेष्टा की गयी है, उतनी ही पूर्ण भाव के प्रकाशन में कठिनाई आई है। वे माया को सत्य नहीं मान सकते इसी से उनका अद्वैतवाद व्यावृत्तिमूलक एवं वैराग्यमूलक है, वह ग्राह्य मूलक नहीं है (कविराज, 1996)। यद्यपि वेदान्त का ब्रह्म सत्, चिद् एवं आनन्द रूप है फिर भी स्वातन्त्र्य रहित होने के कारण शून्य के समकक्ष है। इसलिए परमेश्वर परमशिव ही एकमात्र पूर्ण सत्ता है। वही इस सृष्टि के कण-कण को अपने प्रकाश से अवभासित करते हैं।

इन्दु शर्मा, एम्-फिल्०, पी-एच0 डी०, नेट, असिस्टेन्ट प्रोफेसर, संस्कृत अध्ययन केन्द्र, भाषा विभाग, देव संस्कृति विश्वविद्यालय, हरिद्धार,(उत्तराखण्ड), भारत।

\section{संदर्भ सूची}

ईश्वरकृष्ण (1970). पारिजातकोश- काशी संस्कृत ग्रन्थमाला (पृ० -921) / वाराणसी - चौखम्बा संस्कृत।

कविराज, गोपीनाथ (1996). भारतीय संस्कृति एवं साधना (पृ० 5)। बिहार - राष्ट्रभाषा परिषद्।
तर्कवाचस्पतितारकनाथ (1962). वाचस्पत्यम् (षष्ठ भाग, पृ० 459)। वाराणसी-चौखम्बा संस्कृत ग्रन्थावली। (वस्तु सच्चिदानन्दद्वयं ब्रह्म तथा अज्ञानादि सकलजड़समूहोऽवस्तु "......... ब्रह्मैव नित्यं वस्तु तदन्यखिलमनित्यम्"।)

राधाकान्तदेव (1961). शब्दकलपदुम (तृतीय भाग, पृ० 442-44)। वाराणसी - चौखम्बा संस्कृत ग्रन्थावली।

रस्तोगी, नवजीवन (2002). काश्मीर शैवदर्शन की मूल अवधारणाएँ (पृ० 123)/ नई दिल्ली - मुंशीराम मनोहरलाल प्रकाशन।

Radhakrishana, (1962). Indian Philosophy (Vol.-Il, p. 586). New York: The Macmillan Company.

एकं सद् विप्रा बहुदा वदन्ति (ऋग्वेद संहिता, $1 / 164 / 46)$ ।

केनेषित पतति प्रेषितं मन; केन प्राणः प्रथम: प्रैति युक्तः। केनेषितां वाचमिमां वदन्ति, चक्षु: श्रोत्रं क उदेवो युनक्ति (केनोपनिषद्, 1)।।

आत्मानं ते मनसाऽरादजानाम् (ऋग्वेद संहिता, 1/163/6)।

कतम एको देव इति, प्राण इति, स बह्मत्यदित्याचक्षते (बृहदारण्यकोपनिषद्, 3/9/9)।।

निरतिशयं भूमाख्यं बृहत्वाद् बह्मेति विद्धि (केनोपनिषद्, 1/5)।

ब्रह्मसत्यं जगन्नमिथ्या (तैत्तिरीयोपनिषद्, 2/1/1)।

बृंहति बृंहयति तस्मादुच्यते परं ब्रह्मेति श्रुति (श्वेताश्वतरोपनिषद्, 1/1)।

बृहत्तमत्वाद् बह्मां (तैत्तिरीयोपनिषद्, 2/1/1)।

सत्यं ज्ञानमनन्तं ब्रह्म (तैत्तिरीयोपनिषद्, 2/1/1)।।

न तु तद्द्वितीयमस्तिततोऽन्यद् विभक्तं यत् पश्येत् (बृहदारण्यकोपनिषद्, $3 / 9 / 9)$ ।

सत्यस्थ ब्रह्मणो नाम सत्यमिति (बृहदारण्यकोपनिषद्, 5/5/1)।

तस्मात् सर्वगतं ब्रह्म नित्यं यज्ञ प्रतिष्ठितम् (श्रीमद्भगवद्गीता, 3-15)।

बह्मार्पण बह्म हिवर्बह्माग्नौ बह्मणा हुतम्। बह्मैव तेन गन्तव्यं ब्रह्मकर्मसमाधिना (श्रीमद्भगवद्गीता, 4-24)।।

अस्य जगतोनामरूपाभ्यां.............यत् सर्वज्ञात् सर्वसक्ते: कारणाद् भवति तद् ब्रह्मैव (शारीरकभाष्य, 1/1/2)।।

ब्रह्मावसानोऽयं प्रतिषेधः नाभावावसान. (शारीरकभाष्य, 3/2/22)।।

स: यः सदाख्य एस उक्तोऽणुभावो जगतो मूलम्..........एतेन सदाख्येनात्मनाडऽत्मवत्सर्वमिदं जगत्। येन चात्मनाडुत्मवत्सर्वमिदं जगत् तदैव सदाख्यं कारणं सत्यं परमार्थसत्। अतः स स्वात्मा जगतः प्रत्यक्स्वरूपं सतत्वं याथात्म्यम् (छान्दोग्योपनिषद्, 6/8/7)। 
सम्मूला: सौम्येमा: सर्वा: प्रजाः सदायतनाः सत्प्रतिष्ठाः। यथा सोम्येकेन मृत्पिण्डेन सर्वमृन्मयं विज्ञातं। स्याद् वाचारम्भणं विकारो नामधेयं मृत्तिकेत्येव सत्यम् (छान्दोग्योपनिषद्, 6/1/4)।।

प्रकृतिश्चोपादानकारणं ब्रह्याभ्युपगन्तव्यं निमित्तकारणं च न केवलं निमित्तकारणं (शारीरकभाष्य, 1/4/13)।

कार्यमाकाशादिक बहुप्रप जगत्, कारण परं ब्रह्म (शारीरकभाष्य, 2/12)

यथोर्णनाभिः सृजते गृह्णते च, यथा पृथिव्यामोषधयः सम्भवन्ति। यथा सतः पुरूषात् केशलोमानि, तथाइक्षरात् सम्भवतीह विश्वम् (बृहदारण्यकोपनिषद्, 1/17)।

येनाश्रुतं श्रुतं भवति (छान्दोग्योपनिषद्, $6 / 1 / 13$ )।

यतो वा इमानि भूतानि जायन्ते (तैत्तिरीयोपनिषद्, 2/1/1)।

जगज्जन्मस्थितिलयकारणत्वम् ब्रह्म (शारीरकभाष्य, 1/4/23)।

तथैकस्मिन्नपि ब्रह्मणि स्वरूपानुपमदेनैवानेकाकारा सृष्टिः भवति (तैत्तिरीयोपनिषद्, 2/1/18)।

चितिस्तुर्यातीतपदात्मिका परासंवित् (तंत्रालोकविवेक, भाग-3, पृ० 404)।

प्रकाशमानं न पृथक् प्रकाशात् / स च प्रकाशो न पृथग विमर्शात् (श्रीविज्ञानभैरव, पृ० 44)।

स एव विमृशत्त्वेन नियतेन महेश्वरः (ईश्वरप्रत्यभिज्ञा, 1/8/11)।

न शिव: शक्तिरहितो न शक्तिर्यतिरेकिणी। शिव शक्तस्तथा भावानिच्छया कर्तुमीहते।/ शक्तिशक्तिमतोर्भेदः शैवे जातु न वर्ण्यते (शिवदृष्टि, $3 / 2-3)$ ।

विमर्शो हि सर्वसहः परमपि आत्मीकरोति, आत्मानं च परीकरोति। उभयम् एकीकरोति एकीकृतं द्वयमपिन्यज्भावयति इत्यमेव-स्वभाव: (ईश्वरप्रत्यभिज्ञाविमर्शिनी, भाग-2, पृ० 205)।
स्वतंत्र इति तस्येच्छाशक्तिः स्वातंत्रसंज्ञिता (मालिनीविजवार्तिक, $1 / 87)$ ।

विमर्श एवं देवस्य शुद्धे ज्ञानकिये यतः (ईश्वरप्रत्यभिज्ञाविमर्शिनी, $1 / 8 / 11)$ ।

परामर्शो हि चिकीर्षारूपेच्छा, तस्यां च सर्वमन्तर्भूत निर्मातव्यमभेदकल्पेनास्ते (ईश्वरप्रत्यभिज्ञाविमर्शिनी, भाग-2, पृ० 181)।

आत्मैव सर्वभावेषु स्फुरन्निवृत्तचिद्धपुः। अनिरूद्धेच्छाप्रसरः प्रसरद्दृकक्रिय: शिव: (शिवदृष्टि, 1/2)।।

क्रियाशक्तरेव स्वातंत्र्यामर्शरूपाया: अयं सर्वो विस्फार: (ईश्वरप्रत्यभिज्ञाविमर्शिनी, भाग-2, पृ० 42)।

अयं विश्वमयत्वेडविश्वोत्तीर्णरूतुदुत्तीर्णत्वेडपि तन्मयः (तंत्रालोकविवेक, भाग-3, पृ० 404)।

चितिः स्वतन्त्रा: विश्वसिद्धिहततुः (प्रत्यभिज्ञाहृदयम्, सूत्र-1)।

स्वेच्छया स्वाभित्रौ विश्वमुन्मीलीयति (प्रत्यभिज्ञाहृदयम्, सूत्र-2)।

प्रकाशरूपता चिच्छक्तिः। स्वातन्त्रम् आनन्दशक्तिः। तच्चमत्कार इच्छाशक्तिः। आमर्शात्मकता ज्ञानशक्तिः// सर्वाकारयोगित्वं कियाशक्ति: (तंत्रसार, पृ० 6)।

तेन भगवता......तथा संवित्तावपीति। अतःसर्वमेवैतदाभासमात्रसार मैवेति (तंत्रालोकविवेक, भाग-2, पृ० 29-30)।

सदा सृष्टिविनोदाय सदास्थितिसुखासिने। सदा त्रिभुवनाहारतृप्ताय भवते नम: (ईश्वरप्रत्यभिज्ञाविमर्शिनी, भाग-1, पृ० 195)।

उक्ता सर्वे च विश्रान्तिः सवापेक्षानिरोधतः। स्वातन्त्रमथकर्तृत्वं मुख्यमीश्वातापि च (अजडप्रमातृसिद्धि, भलोक-22)।

तदेव तस्य स्वातन्त्र्यं शक्तिर्नियतिनामिका। यया रूद्र: पशुर्जातु स्वातन्त्र्यं नैव विन्दति (तंत्रालोक आ0-1/222)। 